\title{
On stability of ground states for finite crystals in the Schrödinger-Poisson model
}

\author{
A. Komech ${ }^{1}$ \\ Faculty of Mathematics of Vienna University \\ and Institute for Information Transmission Problems RAS \\ e-mail: alexander.komech@univie.ac.at \\ E. Kopylova ${ }^{2}$ \\ Faculty of Mathematics of Vienna University \\ and Institute for Information Transmission Problems RAS \\ e-mail: elena.kopylova@univie.ac.at
}

\begin{abstract}
We consider the Schrödinger-Poisson-Newton equations for finite crystals under periodic boundary conditions with one ion per cell of a lattice. The electrons are described by one-particle Schrödinger equation.

Our main results are i) the global dynamics with moving ions; ii) the orbital stability of periodic ground state under a novel Jellium and Wiener-type conditions on the ion charge density. Under the Jellium condition both ionic and electronic charge densities for the ground state are uniform.
\end{abstract}

Key words and phrases: crystal; lattice; Schrödinger-Poisson equations; ground state; stability; orbital stability; Hamilton structure; energy conservation; charge conservation; $U(1)$-invariance; Hessian; Fourier transform.

AMS subject classification: 35L10, 34L25, 47A40, 81U05

\footnotetext{
${ }^{1}$ Supported partly by Austrian Science Fund (FWF): P28152-N35, and the grant of RFBR 16-01-00100.

${ }^{2}$ Supported partly by Austrian Science Fund (FWF): P27492-N25, and the grant of RFBR 16-01-00100.
} 


\section{Introduction}

The first mathematical results on the stability of matter were obtained by Dyson and Lenard in $[11,12]$ where the energy bound from below was established. The thermodynamic limit for the Coulomb systems was studied first by Lebowitz and Lieb [23, 24], see the survey and further development in [27]. These results were extended by Catto, Le Bris, Lions and others to Thomas-Fermi and HartreeFock models $[7,8,9]$. Further results in this direction were established by Cancés, Lahbabi, Lewin, Sabin, Stoltz, and others $[5,6,22,25,26]$. All these results concern either the convergence of the ground state of finite particle systems in the thermodynamic limit or the existence of the ground state for infinite particle systems.

However, the dynamical stability of crystals with moving ions was never considered previously. This stability is necessary for a rigorous analysis of fundamental quantum phenomena in the solid state physics: heat conductivity, electric conductivity, thermoelectronic emission, photoelectric effect, Compton effect, etc., see [3].

In present paper we consider the coupled Schrödinger-Poisson-Newton equations for finite crystals under periodic boundary conditions with one ion per cell of a lattice. We construct the global dynamics of crystals with moving ions and prove the conservation of energy and charge.

Our main result is the orbital stability of every ground state with periodic arrangement of ions under novel 'Jellium' and Wiener-type conditions on the ion charge density.

The electron cloud is described by one-particle Schrödinger equation. The ions are described as classical particles that corresponds to the Born and Oppenheimer approximation. The ions interact with the electron cloud via the scalar potential, which is a solution to the corresponding Poisson equation.

This model does not respect the Pauli exclusion principle for electrons. However, it provides a convenient framework to introduce suitable functional tools that might be useful for physically more realistic models (Thomas-Fermi, Hartree-Fock, and second quantized models). In particular, we find a novel stability criterion (1.11), (1.13).

We consider crystals which occupy the finite torus $T_{N}:=\mathbb{R}^{3} / N \mathbb{Z}^{3}$ and have one ion per cell of the cubic lattice $\Gamma_{N}:=\mathbb{Z}^{3} / N \mathbb{Z}^{3}$, where $N \in \mathbb{N}$. The cubic lattice is chosen for the simplicity of notations. We denote by $\sigma(x)$ the charge density of one ion,

$$
\sigma \in C^{2}\left(T_{N}\right), \quad \int_{T_{N}} \sigma(x) d x=e Z>0
$$

where $e>0$ is the elementary charge. Let $\psi(x, t)$ be the wave function of the electron field, $q(n, t)$ denotes the ion displacement from the reference position $n \in \Gamma_{N}$, and $\Phi(x)$ be the electrostatic potential generated by the ions and electrons. We assume $\hbar=c=\mathrm{m}=1$, where $c$ is the speed of light and $\mathrm{m}$ is the electron mass. Then the considered coupled equations read

$$
\begin{aligned}
i \partial_{t} \psi(x, t) & =-\frac{1}{2} \Delta \psi(x, t)-e \Phi(x, t) \psi(x, t), \quad x \in T_{N}, \\
-\Delta \Phi(x, t) & =\rho(x, t):=\sum_{n \in \Gamma_{N}} \sigma(x-n-q(n, t))-e|\psi(x, t)|^{2}, \quad x \in T_{N}, \\
M \ddot{q}(n, t) & =-(\nabla \Phi(x, t), \sigma(x-n-q(n, t))), \quad n \in \Gamma_{N} .
\end{aligned}
$$

Here the brackets $(\cdot, \cdot)$ stand for the scalar product on the real Hilbert space $L^{2}\left(T_{N}\right)$ and for its different extensions, and $M>0$ is the mass of one ion. All derivatives here and below are understood in the 
sense of distributions. Similar finite periodic approximations of crystals are treated in all textbooks on quantum theory of solid state $[4,17,30]$. However, the stability of ground states in this model was never discussed.

Obviously,

$$
\int_{T_{N}} \rho(x, t) d x=0
$$

by the Poisson equation (1.3). Hence, the potential $\Phi(x, t)$ can be eliminated from the system (1.2) (1.4) using the operator $G:=(-\Delta)^{-1}$, see (2.1) for a more precise definition. Substituting $\Phi(\cdot, t)=$ $G \rho(\cdot, t)$ into equations (1.2) and (1.4), we can write the system as

$$
\dot{X}(t)=F(X(t)), \quad t \in \mathbb{R},
$$

where $X(t)=(\psi(\cdot, t), q(\cdot, t), p(\cdot, t))$ with $p(\cdot, t):=\dot{q}(\cdot, t)$. The system (1.2) - (1.4) is equivalent, up to a gauge transform (see the next section), to equation (1.6) with the normalization

$$
\|\psi(\cdot, t)\|_{L^{2}\left(T_{N}\right)}^{2}=Z N^{3}, \quad t \in \mathbb{R}
$$

which follows from (1.5). If the integral (1.1) vanishes, we have $Z=0$ and $\psi(x, t) \equiv 0$.

We will identify the complex functions $\psi(x)$ with two real functions $\psi_{1}(x):=\operatorname{Re} \psi(x)$ and $\psi_{2}(x):=$ $\operatorname{Im} \psi(x)$. Now equation (1.6) can be written as the Hamilton system

$$
\partial_{t} \psi_{1}(x, t)=\frac{1}{2} \partial_{\psi_{2}(x)} E, \partial_{t} \psi_{2}(x, t)=-\frac{1}{2} \partial_{\psi_{1}(x)} E, \partial_{t} q(n, t)=\partial_{p(n)} E, \partial_{t} p(n, t)=-\partial_{q(n)} E .
$$

Here the Hamilton functional (energy) reads

$$
E(\psi, q, p)=\frac{1}{2} \int_{T_{N}}|\nabla \psi(x)|^{2} d x+\frac{1}{2}(\rho, G \rho)+\sum_{n \in \Gamma_{N}} \frac{p^{2}(n)}{2 M}
$$

where $q:=\left(q(n): n \in \Gamma_{N}\right) \in\left[T_{N}\right]^{\bar{N}}, p:=\left(p(n): n \in \Gamma_{N}\right) \in \mathbb{R}^{3 \bar{N}}$ with $\bar{N}:=N^{3}$, and

$$
\rho(x):=\sum_{n \in \Gamma_{N}} \sigma(x-n-q(n))-e|\psi(x)|^{2}, \quad x \in T_{N} .
$$

Our main goal is the stability of ground states, i.e., solutions to (1.2) - (1.4) with minimal (zero) energy (1.9). We will consider only $\Gamma_{N}$-periodic ground states (nonperiodic ground states exist for some degenerate densities $\sigma$, see Remark 1.2 ii) and Section B.3).

We will see that all these $\Gamma_{N}$-periodic ground states can be stable depending on the choice of the ion density $\sigma$. However, we study very special densities $\sigma$ satisfying some conditions below. Namely, we will assume the following condition on the ion charge density,

$$
\text { The Jellium Condition: } \quad \hat{\sigma}(\xi):=\int_{T_{N}} e^{i \xi x} \sigma(x) d x=0, \quad \xi \in \Gamma_{1}^{*} \backslash 0,
$$

where $\Gamma_{1}^{*}:=2 \pi \mathbb{Z}^{3}$. This condition immediately implies that the periodized ion charge density is a positive constant everywhere on the torus:

$$
\sum_{n \in \Gamma_{N}} \sigma(x-n) \equiv e Z, \quad x \in T_{N}
$$


The simplest example of such a $\sigma$ is a constant over the unit cell of a given lattice, which is what physicists usually call Jellium [14]. We give further examples in Section B.2. Here we study this model in the rigorous context of the Schrödinger-Poisson equations.

Furthermore, we will assume a spectral property of the Wiener type

$$
\text { The Wiener Condition: } \Sigma(\theta):=\sum_{m \in \mathbb{Z}^{3}}\left[\frac{\xi \otimes \xi}{|\xi|^{2}}|\hat{\sigma}(\xi)|^{2}\right]_{\xi=\theta+2 \pi m}>0, \quad \theta \in \Pi_{N}^{*} \backslash \Gamma_{1}^{*} \text {, }
$$

where the Brillouin zone $\Pi_{N}^{*}$ is defined by

$$
\Pi_{N}^{*}:=\left\{\xi=\left(\xi^{1}, \xi^{2}, \xi^{3}\right) \in \Gamma_{N}^{*}: 0 \leq \xi^{j} \leq 1, \quad j=1,2,3\right\}, \quad \Gamma_{N}^{*}:=\frac{2 \pi}{N} \mathbb{Z}^{3} .
$$

This condition is an analog of the Fermi Golden Rule for crystals. It is independent of (1.11). We have introduced conditions of type (1.11) and (1.13) in [21] in the framework of infinite crystals.

Remark 1.1. i) The series (1.13) converges for $\theta \in \Gamma_{N}^{*} \backslash \Gamma_{1}^{*}$ by the Parseval identity since $\sigma \in L^{2}\left(T_{N}\right)$ by (1.1).

ii) The matrix $\Sigma(\theta)$ is $\Gamma_{1}^{*}$-periodic outside $\Gamma_{1}^{*}$. Thus, (1.13) means that $\Sigma(\theta)$ is a positive matrix for $\theta \in \bar{\Pi}_{N}^{*} \backslash 0$, where $\bar{\Pi}_{N}^{*}$ is the 'discrete torus' $\Gamma_{N} / \Gamma_{1}^{*}$.

The series (1.13) is a nonnegative matrix. Hence, the Wiener condition holds 'generically'. For example it holds if

$$
\hat{\sigma}(\xi) \neq 0, \quad \xi \in \Gamma_{N}^{*} \backslash \Gamma_{1}^{*},
$$

i.e., (1.11) are the only zeros of $\hat{\sigma}(\xi)$. However, (1.13) does not hold for the simplest Jellium model, when $\sigma$ is constant on the unit cell, see (B.5) and (B.6).

The energy (1.9) is nonnegative, and its minimum is zero. We show in Lemma B.1 that under Jellium condition (1.11) all $\Gamma_{N}$-periodic ground states are zero energy stationary solutions of the form

$$
S_{\alpha, r}=\left(\psi_{\alpha}, \bar{r}, 0\right), \quad \alpha \in[0,2 \pi], \quad r \in T_{N},
$$

where $\psi_{\alpha}(x) \equiv e^{i \alpha} \sqrt{Z}$ and $\bar{r} \in\left[T_{N}\right]^{\bar{N}}$ is defined by

$$
\bar{r}(n)=r, \quad n \in \Gamma_{N} .
$$

The corresponding electronic charge density reads

$$
\rho^{e}(x):=-e\left|\psi_{\alpha}(x)\right|^{2} \equiv-e Z, \quad x \in T_{N}
$$

Hence, the corresponding total charge density (1.10) identically vanishes by (1.12). Let us emphasize that both ionic and electronic charge densities are uniform for the ground state under the Jellium condition.

Our main result (Theorem 4.7) is the stability of the real 4-dimensional 'solitary manifold'

$$
\mathscr{S}=\left\{S_{\alpha, r}: \alpha \in[0,2 \pi], r \in T_{N}\right\} .
$$

The stability means that any solution $X(t)=(\psi(\cdot, t), q(\cdot, t), p(\cdot, t))$ to (1.6) with initial data, lying in the vicinity of the manifold $\mathscr{S}$, is close to it uniformly in time. This is the 'orbital stability' in the 
sense of [15], since the manifold $\mathscr{S}=S^{1} \times T_{N} \times\{0\}$ is the orbit of the symmetry group $U(1) \times T_{N}$. Obviously,

$$
E(S)=0, \quad S \in \mathscr{S} .
$$

Let us comment on our approach. We prove the local well-posedness for the system (1.2) - (1.4) by the contraction mapping principle. The global well-posedness for the equation (1.6) and the charge and energy conservation follow by the Galerkin approximations and the uniqueness of solutions. We apply the charge conservation to return back to the system (1.2) - (1.4).

The orbital stability of the solitary manifold $\mathscr{S}$ is deduced from the lower energy estimate

$$
E(X) \geq v d^{2}(X, \mathscr{S}) \quad \text { if } \quad d(X, \mathscr{S}) \leq \delta, \quad X \in \mathscr{M}
$$

where $\mathscr{M}$ is the manifold defined by the normalization (1.7) (see Definition 4.4); $v, \delta>0$ and ' $d$ ' is the distance in the 'energy norm'. This estimate obviously implies the stability of the solitary manifold $\mathscr{S}$. We deduce (1.21) from the positivity of the Hessian $E^{\prime \prime}(S)$ for $S \in \mathscr{S}$ in the orthogonal directions to $\mathscr{S}$ on the manifold $\mathscr{M}$. The Jellium and Wiener conditions are sufficient for this positivity. We expect that these conditions are also necessary; however, this is still an open problem. Anyway, the positivity can break down when these conditions fail. We have shown this in [21, Lemma 10.1] in the context of infinite crystals, however the proof extends directly to the finite crystals. The Jellium condition cancels the negative energy which is provided by the electrostatic instability ('Earnshaw's Theorem' [29], see [21, Remark 10.2]).

Our main novelties are the following.

I. The well-posedness and the energy and charge conservation for the system (1.2) - (1.4).

II. The calculation of all ground states; in particular, the existence of ground states with periodic and with non-periodic ion arrangements.

III. The orbital stability of $\Gamma_{N}$-periodic ground states.

IV. The lower energy estimate (1.21).

Remarks 1.2. i) In the case of infinite crystal, corresponding to $N=\infty$, the orbital stability seems impossible. Namely, for $N=\infty$ the estimates (3.5), (4.37) and (4.40) break down, as well as the estimate of type (1.21) which is due to the discrete spectrum of the energy Hessian $E^{\prime \prime}(S)$ on the compact torus.

ii) We show that the identity of type (1.12) holds for a wide set of arrangements of ions which are not $\Gamma_{1}$-periodic, if $\sigma$ satisfy additional spectral conditions. The corresponding examples are given, but in all our examples the Wiener condition breaks down. We suppose that the Wiener condition provides the periodicity (1.17), however this is a challenging open problem, see Section B.4. We prove the orbital stability only for $\Gamma_{1}$-periodic ground states.

iii) The extension of our results to the Hartree-Fock model is not straightforward. Even the existance of solutions requires quite novel ideas as well as the calculation of the null space of the Hessian.

Let us comment on previous works in this direction.

The ground state for crystals in the Schrödinger-Poisson model was constructed in $[19,20]$, and its linear stability was proved in [21].

In the Hartree-Fock model the crystal ground state was constructed for the first time by Catto, Le Bris, and Lions [8, 9]. For the Thomas-Fermi model, see [7]. 
In [6], Cancés and Stoltz have established the well-posedness for the dynamics of local perturbations of the crystal ground state in the random phase approximation for the reduced Hartree-Fock equations with the Coulomb pairwise interaction potential $w(x-y)=1 /|x-y|$. The space-periodic nuclear potential in the equation [6, (3)] does not depend on time that corresponds to the fixed nuclei positions. The nonlinear Hartree-Fock dynamics for crystals with the Coulomb potential and without the random phase approximation was not studied previously, see the discussion in [22] and in the introductions of $[5,6]$.

In [5] E. Cancès, S. Lahbabi, and M. Lewin have considered the random reduced HF model of crystal when the ions charge density and the electron density matrix are random processes, and the action of the lattice translations on the probability space is ergodic. The authors obtain suitable generalizations of the Hoffmann-Ostenhof and Lieb-Thirring inequalities for ergodic density matrices, and construct a random potential which is a solution to the Poisson equation with the corresponding stationary stochastic charge density. The main result is the coincidence of this model with the thermodynamic limit in the case of the short range Yukawa interaction.

In [25], Lewin and Sabin have established the well-posedness for the reduced von Neumann equation, describing the Fermi gas, with density matrices of infinite trace and pair-wise interaction potentials $w \in L^{1}\left(\mathbb{R}^{3}\right)$. Moreover, the authors prove the asymptotic stability of translation-invariant stationary states for 2D Fermi gas [26].

The paper is organized as follows. In Section 2 we eliminate the potential and reduce the dynamics to the integral equation. In Sections 3 we prove the well-posedness. In Section 4 we prove the stability of the solitary manifold $\mathscr{S}$ establishing the lower estimate for the energy. In Appendices we prove the conservation of the energy and charge, describe all ground states and give some examples.

Acknowledgments The authors are grateful to Herbert Spohn for helpful discussions and remarks.

\section{Reduction to the integral equation}

The operator $G:=(-\Delta)^{-1}$ is well defined in the Fourier series:

$$
\rho(x)=\sum_{\xi \in \Gamma_{N}^{*}} \hat{\rho}(\xi) e^{i \xi x}, \quad G \rho:=\sum_{\xi \in \Gamma_{N}^{*} \backslash 0} \frac{\hat{\rho}(\xi)}{\xi^{2}} e^{i \xi x} .
$$

The Poisson equation (1.3) implies that $\hat{\rho}(0, t)=\int \rho(x, t) d x=0$, which is equivalent to (1.7). Hence, $\Phi(\cdot, t)=G \rho(\cdot, t)$ up to an additive constant $C(t)$ which can be compensated by a gauge transform $\psi(x, t) \mapsto \psi(x, t) \exp \left(i e \int_{0}^{t} C(s) d s\right)$. The system (1.8) can be written as

$$
\dot{X}(t)=J E^{\prime}(X(t)), \quad X(t):=\left(\psi_{1}(t)+i \psi_{2}(t), q(t), p(t)\right),
$$

where

$$
J=\left(\begin{array}{rrr}
-i / 2 & 0 & 0 \\
0 & 0 & 1 \\
0 & -1 & 0
\end{array}\right) .
$$

We will use the following function spaces with $s=0, \pm 1$. Let us define the Sobolev space $H^{s}\left(T_{N}\right)$ as real Hilbert spaces of complex-valued functions with the scalar product

$$
(\psi, \varphi)_{s}:=\operatorname{Re} \int_{T_{N}} \sum_{|\alpha| \leq s} \partial^{\alpha} \psi(x) \partial^{\alpha} \bar{\varphi}(x) d x, \quad s=0,1 .
$$


By definition, $H^{-1}\left(T_{N}\right)$ is the real dual space to $H^{1}\left(T_{N}\right)$ which will be identified with distributions by means of the scalar product in $H^{0}\left(T_{N}\right)$.

Definition 2.1. i) $\mathscr{W}^{s}$ denotes the real Hilbert space $H^{s}\left(T_{N}\right) \oplus \mathbb{R}^{3 \bar{N}} \oplus \mathbb{R}^{3 \bar{N}}$ for $s=0, \pm 1$.

ii) $\mathscr{V}^{s}:=H^{s}\left(T_{N}\right) \times\left[T_{N}\right]^{\bar{N}} \times \mathbb{R}^{3 \bar{N}}$ is the Hilbert manifold endowed with the metric

$$
d_{\mathscr{Y} s}\left(X, X^{\prime}\right):=\left\|\psi-\psi^{\prime}\right\|_{H^{s}\left(T_{N}\right)}+\left|q-q^{\prime}\right|+\left|p-p^{\prime}\right|, \quad X=(\psi, q, p), \quad X^{\prime}=\left(\psi^{\prime}, q^{\prime}, p^{\prime}\right)
$$

and with the 'quasinorm'

$$
|X|_{\mathscr{V}^{s}}:=\|\psi\|_{H^{s}\left(T_{N}\right)}+|p|, \quad X=(\psi, q, p) .
$$

The linear space $\mathscr{W}^{s}$ is isomorphic to the tangent space to the Hilbert manifold $\mathscr{V}^{s}$ at each point $X \in \mathscr{V}^{s}$. We will write $\mathscr{X}:=\mathscr{V}^{0}, \mathscr{V}:=\mathscr{V}^{1}, \mathscr{W}:=\mathscr{W}^{1}$, and $(\cdot, \cdot)_{0}=(\cdot, \cdot)$, which agrees with the definition of the scalar product on the real Hilbert space $L^{2}\left(T_{N}\right)$. In particular,

$$
(1, i)=0 .
$$

Denote by the brackets $\langle\cdot, \cdot\rangle$ the scalar product in $\mathscr{X}$ and also the duality between $\mathscr{W}^{-1}$ and $\mathscr{W}^{1}$ :

$$
\left\langle Y, Y^{\prime}\right\rangle:=\left(\varphi, \varphi^{\prime}\right)+\varkappa \varkappa^{\prime}+\pi \pi^{\prime}, \quad Y=(\varphi, \varkappa, \pi), \quad Y^{\prime}=\left(\varphi^{\prime}, \varkappa^{\prime}, \pi^{\prime}\right) .
$$

The total electronic charge is defined (up to a factor) by

$$
Q(X):=\int|\psi(x)|^{2} d x, \quad X=(\psi, q, p) \in \mathscr{V} .
$$

Obviously,

$$
|X|_{\mathscr{V}}^{2} \leq C[E(X)+Q(X)], \quad X \in \mathscr{V},
$$

The system (2.2) is a nonlinear infinite-dimensional perturbation of the free Schrödinger equation. We will prove that a solution $X \in C(\mathbb{R}, \mathscr{V})$ exists and is unique for any initial state $X(0) \in \mathscr{V}$, and the energy and the electronic charge are conserved,

$$
E(X(t))=E(X(0)), \quad Q(X(t))=Q(X(0)), \quad t \in \mathbb{R} .
$$

The energy (1.9) and the charge are well defined and continuous on $\mathscr{V}$ in the metric $d_{\mathscr{V}}$ by the estimate (3.12) below. The charge conservation holds by the Noether theory $[2,15,18]$ due to the $U(1)$ invariance of the Hamilton functional:

$$
E\left(e^{i \alpha} \psi, q, p\right)=E(\psi, q, p), \quad(\psi, q, p) \in \mathscr{V}, \quad \alpha \in \mathbb{R} .
$$

We rewrite the system (2.2) in the integral form

$$
\left\{\begin{array}{l}
\psi(t)=e^{-i H_{0} t} \psi(0)+i e \int_{0}^{t} e^{-i H_{0}(t-s)}[\Phi(s) \psi(s)] d s \\
q(n, t)=q(n, 0)+\frac{1}{M} \int_{0}^{t} p(n, s) d s \quad \bmod N \mathbb{Z}^{3} \\
p(n, t)=p(n, 0)-\int_{0}^{t}(\nabla \Phi(s), \sigma(\cdot-n-q(n, s))) d s
\end{array}\right.
$$


where $H_{0}:=-\frac{1}{2} \Delta$ and $\Phi(s):=G \rho(s)$. In the vector form (2.13) reads

$$
X(t)=e^{-A t} X(0)+\int_{0}^{t} e^{-A(t-s)} N(X(s)) d s \quad \bmod \left(\begin{array}{c}
0 \\
N \mathbb{Z}^{3} \\
0
\end{array}\right)
$$

Here

$$
A=\left(\begin{array}{ccc}
i H_{0} & 0 & 0 \\
0 & 0 & 0 \\
0 & 0 & 0
\end{array}\right), \quad N(X)=(i e \Phi \psi, p, f), \quad f(n):=-(\nabla \Phi, \sigma(\cdot-n-q(n))), \quad \Phi:=G \rho,
$$

where $\rho$ is defined by (1.10).

\section{Global dynamics}

In this section we prove the well-posedness of the dynamics.

Theorem 3.1. (Global well-posedness). Let (1.1) hold and $X(0) \in \mathscr{V}$. Then

i) Equation (2.2) has a unique solution $X \in C(\mathbb{R}, \mathscr{V})$, and the maps $U(t): X(0) \mapsto X(t)$ are continuous in $\mathscr{V}$ for $t \in \mathbb{R}$.

ii) The conservation laws (2.11) hold.

iii) $X$ is the solution to (1.2) - (1.4) if

$$
Q(X(0))=Z \bar{N}^{3}
$$

First, let us prove the local well-posedness.

Proposition 3.2. (Local well-posedness). Let (1.1) hold and $|X(0)|_{\mathscr{V}} \leq R$. Then there exists $\tau=$ $\tau(R)>0$ such that equation (2.2) has a unique solution $X \in C([-\tau, \tau], \mathscr{V})$, and the maps $U(t)$ : $X(0) \mapsto X(t)$ are continuous in $\mathscr{V}$ for $t \in[-\tau, \tau]$.

In the next two lemmas we prove the boundedness and the local Lipschitz continuity of the nonlinearity $N: \mathscr{V} \rightarrow \mathscr{W}$. With this proviso Proposition 3.2 follows from the integral form (2.14) of the equation (2.2) by the contraction mapping principle, since $e^{-i H t}$ is an isometry of $\mathscr{W}$.

Lemma 3.3. For any $R>0$ and $|X|_{\mathscr{V}} \leq R$

$$
\|N(X)\|_{\mathscr{W}} \leq C(R)
$$

Proof. We split $\rho(x, t)$ as $\rho(x, t)=\rho^{i}(x, t)+\rho^{e}(x, t)$, where

$$
\rho^{i}(x, t)=\sum_{n \in \Gamma_{N}} \sigma(x-n-q(n, t)), \quad \rho^{e}(x, t)=-e|\psi(x, t)|^{2} .
$$

Applying the Cauchy-Schwarz inequality to the second formula (2.1), we obtain for $\Phi:=G \rho$,

$$
\|\Phi\|_{C\left(T_{N}\right)} \leq C\|\hat{\rho}\|_{L^{2}\left(\Gamma_{N}^{*}\right)}=C\|\rho\|_{L^{2}\left(T_{N}\right)} \leq C\left(\left\|\rho^{i}\right\|_{L^{2}\left(T_{N}\right)}+e\|\psi\|_{L^{4}\left(T_{N}\right)}^{2}\right) \leq C_{1}\left(1+\|\psi\|_{H^{1}\left(T_{N}\right)}^{2}\right)
$$


since $H^{1}\left(T_{N}\right) \subset L^{6}\left(T_{N}\right) \subset L^{4}\left(T_{N}\right)$. On the other hand, the Hölder inequality implies that

$$
\left\|\nabla \rho^{e}\right\|_{L^{3 / 2}\left(T_{N}\right)} \leq e\left\|\nabla|\psi|^{2}\right\|_{L^{3 / 2}\left(T_{N}\right)} \leq C_{1}\|\psi\|_{L^{6}\left(T_{N}\right)}\|\nabla \psi\|_{L^{2}\left(T_{N}\right)} \leq C_{2}\|\psi\|_{H^{1}\left(T_{N}\right)}^{2} .
$$

Therefore, we get by the Hausdorff-Young and the Hölder inequalities

$$
\begin{aligned}
\|\nabla \Phi\|_{L^{3}\left(T_{N}\right)} & \leq C\|\widehat{\nabla \Phi}\|_{L^{3 / 2}\left(\Gamma_{N}^{*}\right)} \leq C_{1}\|\xi \hat{\rho}\|_{L^{3}\left(\Gamma_{N}^{*}\right)}\left[\sum_{\xi \in \Gamma_{N}^{*} \backslash 0}|\xi|^{-6}\right]^{1 / 3} \leq C_{2}\|\nabla \rho\|_{L^{3 / 2}\left(T_{N}\right)} \\
& \leq C_{2}\left(\left\|\nabla \rho^{i}\right\|_{L^{3 / 2}\left(T_{N}\right)}+\left\|\nabla \rho^{e}\right\|_{L^{3 / 2}\left(T_{N}\right)} \leq C_{3}\left(1+\|\psi\|_{H^{1}\left(T_{N}\right)}^{2}\right) .\right.
\end{aligned}
$$

Now (3.3) and (3.5) imply by the Hölder inequality

$$
\begin{aligned}
\|\psi \Phi\|_{L^{2}\left(T_{N}\right)} & \leq\|\Phi\|_{C\left(T_{N}\right)} \cdot\|\psi\|_{L^{2}\left(T_{N}\right)} \leq C\left(1+\|\psi\|_{H^{1}\left(T_{N}\right)}^{3}\right) \\
\|\nabla \psi \Phi\|_{L^{2}\left(T_{N}\right)} & \leq\|\Phi\|_{C\left(T_{N}\right)}\|\nabla \psi\|_{L^{2}\left(T_{N}\right)} \leq C\left(1+\|\psi\|_{H^{1}\left(T_{N}\right)}^{3}\right) \\
\|\psi \nabla \Phi\|_{L^{2}\left(T_{N}\right)} & \leq C\|\psi\|_{L^{6}\left(T_{N}\right)} \cdot\|\nabla \Phi\|_{L^{3}\left(T_{N}\right)} \leq C_{1}\left(1+\|\psi\|_{H^{1}\left(T_{N}\right)}^{3}\right) .
\end{aligned}
$$

Hence,

$$
\|\Phi \psi\|_{H^{1}\left(T_{N}\right)} \leq C\left(1+\|\psi\|_{H^{1}\left(T_{N}\right)}^{3}\right) .
$$

Finally, (3.5) and (1.1) imply that

$$
|f(n)| \leq\|\Phi\|_{C\left(T_{N}\right)}\|\nabla \sigma\|_{L^{1}\left(T_{N}\right)} \leq C\left(1+\|\psi\|_{H^{1}\left(T_{N}\right)}^{2}\right), \quad n \in \Gamma_{N} .
$$

At last, (3.2) holds by (3.6) and (3.7).

It remains to prove that the nonlinearity is locally Lipschitz.

Lemma 3.4. For any $R>0$ and $X_{1}, X_{2} \in \mathscr{V}$

$$
\left\|N\left(X_{1}\right)-N\left(X_{2}\right)\right\|_{\mathscr{W}} \leq C^{\prime}(R) d_{\mathscr{V}}\left(X_{1}, X_{2}\right) \quad \text { if } \quad\left|X_{1}\right|_{\mathscr{V}},\left|X_{2}\right|_{\mathscr{V}} \leq R .
$$

Proof. Writing $X_{k}=\left(\psi_{k}, q_{k}, p_{k}\right)$ and $\Phi_{k}=G \rho_{k}$, we obtain that

$$
\left\|\Phi_{1} \psi_{1}-\Phi_{2} \psi_{2}\right\|_{H^{1}\left(T_{N}\right)} \leq\left\|\left(\Phi_{1}-\Phi_{2}\right) \psi_{1}\right\|_{H^{1}\left(T_{N}\right)}+\left\|\Phi_{2}\left(\psi_{1}-\psi_{2}\right)\right\|_{H^{1}\left(T_{N}\right)} .
$$

Similarly to (3.3) - (3.5) we obtain

$$
\begin{aligned}
\left\|\Phi_{2}\left(\psi_{1}-\psi_{2}\right)\right\|_{H^{1}\left(T_{N}\right)} & \leq\left\|\Phi_{2}\right\|_{C\left(T_{N}\right)}\left\|\psi_{1}-\psi_{2}\right\|_{H^{1}\left(T_{N}\right)}+\left\|\nabla \Phi_{2}\right\|_{L^{3}\left(T_{N}\right)}\left\|\psi_{1}-\psi_{2}\right\|_{L^{6}\left(T_{N}\right)} \\
& \leq C\left(1+R^{2}\right)\left\|\psi_{1}-\psi_{2}\right\|_{H^{1}\left(T_{N}\right)} \leq C(R) d_{\mathscr{V}}\left(X_{1}, X_{2}\right)
\end{aligned}
$$

Further, similarly to (3.4),

$$
\left\|\nabla\left(\rho_{1}^{e}-\rho_{2}^{e}\right)\right\|_{L^{3 / 2}\left(T_{N}\right)} \leq C\left\|\psi_{1}-\psi_{2}\right\|_{H^{1}\left(T_{N}\right)}\left[\left\|\psi_{1}\right\|_{H^{1}\left(T_{N}\right)}+\left\|\psi_{2}\right\|_{H^{1}\left(T_{N}\right)}\right] .
$$

Moreover, $|\sigma(x)-\sigma(x-a)| \leq C|a|$, where $|a|:=\min _{r \in a}|r|$ for $a \in T_{N}$. Hence, similarly to (3.5),

$$
\begin{array}{r}
\left\|\left(\Phi_{1}-\Phi_{2}\right) \psi_{1}\right\|_{H^{1}\left(T_{N}\right)} \leq\left\|\Phi_{1}-\Phi_{2}\right\|_{C\left(T_{N}\right)}\left\|\psi_{1}\right\|_{H^{1}\left(T_{N}\right)}+\left\|\nabla\left(\Phi_{1}-\Phi_{2}\right)\right\|_{L^{3}\left(T_{N}\right)}\left\|\psi_{1}\right\|_{L^{6}\left(T_{N}\right)} \\
\leq C R\left[\left\|\rho_{1}^{i}-\rho_{2}^{i}\right\|_{L^{2}\left(T_{N}\right)}+\left\|\rho_{1}^{e}-\rho_{2}^{e}\right\|_{L^{2}\left(T_{N}\right)}+\left\|\nabla\left(\rho_{1}^{i}-\rho_{2}^{i}\right)\right\|_{L^{3 / 2}\left(T_{N}\right)}+\left\|\nabla\left(\rho_{1}^{e}-\rho_{2}^{e}\right)\right\|_{L^{3 / 2}\left(T_{N}\right)}\right] \\
\leq C_{1} R\left(\left|q_{1}-q_{2}\right|+R\left\|\psi_{1}-\psi_{2}\right\|_{H^{1}\left(T_{N}\right)}\right) \leq C(R) d_{\mathscr{V}}\left(X_{1}, X_{2}\right) .
\end{array}
$$


Now (3.9) and (3.10) give

$$
\left\|\Phi_{1} \psi_{1}-\Phi_{2} \psi_{2}\right\|_{H^{1}\left(T_{N}\right)} \leq C(R) d_{\mathscr{V}}\left(X_{1}, X_{2}\right)
$$

Similarly,

$$
\begin{aligned}
& \left|\left(\nabla \Phi_{1}, \sigma\left(\cdot-n-q_{1}(n)\right)\right)-\left(\nabla \Phi_{2}, \sigma\left(\cdot-n-q_{2}(n)\right)\right)\right| \\
& \leq\left|\left(\nabla \Phi_{1}-\nabla \Phi_{2}, \sigma\left(\cdot-n-q_{1}(n)\right)\right)\right|+\left|\left(\nabla \Phi_{2}, \sigma\left(\cdot-n-q_{1}(n)\right)-\sigma\left(\cdot-n-q_{2}(n)\right)\right)\right| \\
& \leq C\left(\left\|\Phi_{1}-\Phi_{2}\right\|_{C\left(T_{N}\right)}+\left\|\Phi_{2}\right\|_{C\left(T_{N}\right)}\left|q_{1}-q_{2}\right| \leq C(R) d_{\mathscr{V}}\left(X_{1}, X_{2}\right) .\right.
\end{aligned}
$$

This estimate and (3.12) imply (3.8).

Now Proposition 3.2 follows from Lemmas 3.3 and 3.4.

Proof of Theorem 3.1. The local solution $X \in C([-\tau, \tau], \mathscr{V})$ exists and is unique by Proposition 3.2. On the other hand, the conservation laws (2.11) (proved in Proposition A.2 iii)) together with (2.10) imply a priori bound

$$
|X(t)|_{\mathscr{V}}^{2} \leq C[E(X(0))+Q(X(0))], \quad t \in[-\tau, \tau] .
$$

Hence, the local solution admits an extension to the global one $X \in C(\mathbb{R}, \mathscr{V})$. Further, (3.1) implies that $Q(X(t))=Z \bar{N}^{3}$ for all $t \in \mathbb{R}$ by the charge conservation (2.11). Hence, (2.2) gives (1.2) - (1.4).

\section{The orbital stability of the ground state}

In this section we expand the energy into the Taylor series and prove the orbital stability checking the positivity of the energy Hessian.

\subsection{The Taylor expansion of the Hamilton functional}

We will deduce the lower estimate (1.21) using the Taylor expansion of $E(S+Y)$ for $S=S_{\alpha, r}:=$ $\left(\psi_{\alpha}, \bar{r}, 0\right) \in \mathscr{S}$ and $Y=(\varphi, \varkappa, p) \in \mathscr{W}$ :

$$
E(S+Y)=E(S)+\left\langle E^{\prime}(S), Y\right\rangle+\frac{1}{2}\left\langle Y, E^{\prime \prime}(S) Y\right\rangle+R(S, Y)=\frac{1}{2}\left\langle Y, E^{\prime \prime}(S) Y\right\rangle+R(S, Y)
$$

since $E(S)=0$ and $E^{\prime}(S)=0$. First, we expand the charge density (1.10) corresponding to $S+Y=$ $\left(\psi_{\alpha}+\varphi, \bar{r}+\varkappa, p\right)$ :

$$
\rho(x)=\rho^{(0)}(x)+\rho^{(1)}(x)+\rho^{(2)}(x), \quad x \in T_{N},
$$

where $\rho^{(0)}$ and $\rho^{(1)}$ are respectively the terms of zero and first order in $Y$, while $\rho^{(2)}$ is the remainder. However, $\rho^{(0)}(x)$ is the total charge density of the ground state which is identically zero by (1.12) and (1.18):

$$
\rho^{(0)}(x)=\rho_{0}^{i}(x)-e\left|\psi_{\alpha}(x)\right|^{2} \equiv 0, \quad x \in T_{N} .
$$

Thus, $\rho=\rho^{(1)}+\rho^{(2)}$. Expanding (1.10) further, we obtain

$$
\begin{aligned}
& \rho^{(1)}(x)=\sigma^{(1)}(x)-2 e \psi_{\alpha}(x) \cdot \varphi(x), \quad \sigma^{(1)}(x)=-\sum_{n \in \Gamma_{N}} \varkappa(n) \cdot \nabla \sigma(x-n-r), \\
& \rho^{(2)}(x)=\sigma^{(2)}(x)-e|\varphi(x)|^{2}, \quad \sigma^{(2)}(x)=\frac{1}{2} \sum_{n \in \Gamma_{N}} \int_{0}^{1}(1-s)[\varkappa(n) \cdot \nabla]^{2} \sigma(x-n-r-s \varkappa(n)) d s .
\end{aligned}
$$


Substituting $\psi=\psi_{\alpha}+\varphi$ and $\rho=\rho^{(1)}+\rho^{(2)}$ into (1.9), we obtain that the quadratic part of (4.1) reads

$$
\frac{1}{2}\left\langle Y, E^{\prime \prime}(S) Y\right\rangle=\frac{1}{2} \int_{T_{N}}|\nabla \varphi(x)|^{2} d x+\frac{1}{2}\left(\rho^{(1)}, G \rho^{(1)}\right)+K(p), \quad K(p):=\sum_{n} \frac{p^{2}(n)}{2 M}
$$

and the remainder equals

$$
R(S, Y)=\frac{1}{2}\left(2 \rho^{(1)}+\rho^{(2)}, G \rho^{(2)}\right)
$$

\subsection{The null space of the energy Hessian}

In this section we calculate the null space

$$
\mathscr{K}(S):=\left.\operatorname{Ker} E^{\prime \prime}(S)\right|_{\mathscr{W}}, \quad S \in \mathscr{S}
$$

under the Wiener condition.

Lemma 4.1. Let the Jellium and the Wiener conditions (1.11), (1.13) hold and $S \in \mathscr{S}$. Then

$$
\mathscr{K}(S)=\left\{(C, \bar{s}, 0): C \in \mathbb{C}, s \in \mathbb{R}^{3}\right\},
$$

where $\bar{s} \in \mathbb{R}^{3 \bar{N}}$ is defined similarly to (1.17): $\bar{s}(n) \equiv s$.

Proof. All summands o of the energy (4.6) are nonnegative. Hence, this expression is zero if and only if all the summands vanish:

$$
\varphi(x) \equiv C, \quad\left(\rho^{(1)}, G \rho^{(1)}\right)=\left\|\sqrt{G}\left[\sigma^{(1)}-2 e \psi_{\alpha} \cdot \varphi\right]\right\|_{L^{2}\left(T_{N}\right)}^{2}=0, \quad p=0 .
$$

Note that $\sqrt{G} \psi_{\alpha} \cdot \varphi=\sqrt{G} \psi_{\alpha} \cdot C=0$ since the operator $G$ annihilates the constant functions by (2.1). Hence, (4.10) implies that

$$
\sqrt{G} \sigma^{(1)}=0 .
$$

On the other hand, (4.4) gives in the Fourier transform

$$
\hat{\sigma}^{(1)}(\xi)=\hat{\sigma}(\xi) \xi \cdot \sum_{n \in \Gamma_{N}} i e^{i \xi(n+r)} \varkappa(n)=i \hat{\sigma}(\xi) \xi \cdot e^{i \xi r} \hat{\varkappa}(\xi), \quad \xi \in \Gamma_{N}^{*}
$$

where $\hat{\varkappa}(\xi):=\sum_{n \in \Gamma_{N}} i e^{i \xi n} \varkappa(n)$ is a $2 \pi \mathbb{Z}^{3}$-periodic function on $\Gamma_{N}^{*}$. Hence, definition (2.1) and the Jellium condition (1.11) imply that

$$
\begin{aligned}
0=\left\|\sqrt{G} \sigma^{(1)}\right\|_{L^{2}\left(T_{N}\right)}^{2} & =N^{-3} \sum_{\Gamma_{N}^{*} \backslash \Gamma_{1}^{*}}\left|\hat{\sigma}(\xi) \frac{\xi \hat{\varkappa}(\xi)}{|\xi|}\right|^{2} \\
& =N^{-3} \sum_{\theta \in \Pi_{N}^{*} \backslash \Gamma_{1}^{*}}\left\langle\hat{\varkappa}(\theta), \sum_{m \in \mathbb{Z}^{3}}\left[\frac{\xi \otimes \xi}{|\xi|^{2}}|\hat{\sigma}(\xi)|^{2}\right]_{\xi=\theta+2 \pi m} \hat{\varkappa}(\theta)\right\rangle \\
& =N^{-3} \sum_{\theta \in \Pi_{N}^{*} \backslash \Gamma_{1}^{*}}\langle\hat{\varkappa}(\theta), \Sigma(\theta) \hat{\varkappa}(\theta)\rangle .
\end{aligned}
$$

As a result,

$$
\hat{\varkappa}(\theta)=0, \quad \theta \in \Pi_{N}^{*} \backslash \Gamma_{1}^{*}
$$

by the Wiener condition (1.13). On the other hand, $\hat{\varkappa}(0) \in \mathbb{R}^{3}$ remains arbitrary, see Remark 1.1 ii). Respectively, $\varkappa=\bar{s}$ with an arbitrary $s \in \mathbb{R}^{3}$. 
Remark 4.2. The key point of the proof is the explicit calculation (4.12) in the Fourier transform. This calculation relies on the invariance of the Hessian $E^{\prime \prime}(S)$ with respect to $\Gamma_{N}$-translations which is due to the periodicity of the ions arrangement of the ground state.

Remark 4.3. (Beyond the Wiener condition.) If the Wiener condition (1.13) fails, the dimension of the space

$$
V:=\left\{v \in \mathbb{R}^{3 \bar{N}}: \quad v(n)=\sum_{\theta \in \Pi_{N}^{*} \backslash \Gamma_{1}^{*}} e^{-i \theta n} \hat{v}(\theta), \quad \hat{v}(\theta) \in \mathbb{C}^{3}, \Sigma(\theta) \hat{v}(\theta)=0\right\}
$$

is positive. The above calculations show that in this case

$$
\mathscr{K}(S)=\left\{(C, \bar{s}+v, 0): C \in \mathbb{C}, s \in T_{N}, v \in V\right\} .
$$

The subspace $V \subset \mathbb{R}^{3 \bar{N}}$ is orthogonal to the $3 D$ subspace $\left\{\bar{s}: s \in \mathbb{R}^{3}\right\} \subset \mathbb{R}^{3 \bar{N}}$ by the Parseval theorem. Hence, $\operatorname{dim} \mathscr{K}(S)=5+d$, where $d:=\operatorname{dim} V>0$. Thus, $\operatorname{dim} \mathscr{K}(S)>5$. Under the Wiener condition $V=0$, and (4.16) coincides with (4.9).

\subsection{The positivity of the Hessian}

Denote by $N_{S} \mathscr{S}$ the normal subspace to $\mathscr{S}$ at a point $S$ :

$$
N_{S} \mathscr{S}:=\left\{Y \in \mathscr{W}:\langle Y, \tau\rangle=0, \tau \in T_{S} \mathscr{S}\right\}
$$

where $T_{S} \mathscr{S}$ is the tangent space to $\mathscr{S}$ at the point $Y$ and $\langle\cdot, \cdot\rangle$ stands for the scalar product (2.8).

Definition 4.4. Denote by $\mathscr{M}$ the Hilbert manifold

$$
\mathscr{M}:=\left\{X \in \mathscr{V}: Q(X)=Z N^{3}\right\}
$$

Obviously, $\mathscr{S} \subset \mathscr{M}$, and a tangent space to $\mathscr{M}$ at a point $S=\left(\psi_{\alpha}, \bar{r}, 0\right)$ is given by

$$
T_{S} \mathscr{M}=\left\{(\varphi, \varkappa, \pi) \in \mathscr{W}: \varphi \perp \psi_{\alpha}, \varkappa \in \mathbb{R}^{3 \bar{N}}, \pi \in \mathbb{R}^{3 \bar{N}}\right\}
$$

since $D Q\left(\psi_{\alpha}, \bar{r}, 0\right)=\left(\psi_{\alpha}, 0,0\right)$.

Lemma 4.5. Let the Jellium condition (1.11) hold and $S=S_{\alpha, r} \in \mathscr{S}$. Then the Wiener condition (1.13) is necessary and sufficient for the positivity of the Hessian $E^{\prime \prime}(S)$ in the orthogonal directions to $\mathscr{S}$ on $\mathscr{M}$, i.e.,

$$
\left.E^{\prime \prime}(S)\right|_{N_{S} \mathscr{S} \cap T_{S} \mathscr{M}}>0
$$

Proof. i) Sufficiency. Differentiating $S_{\alpha, r}=\left(e^{i \alpha} \psi_{0}, \bar{r}, 0\right) \in \mathscr{S}$ in the parameters $\alpha \in[0,2 \pi]$ and $r \in$ $T_{N}$, we obtain

$$
T_{S} \mathscr{S}=\left\{\left(i C \psi_{\alpha}, \bar{s}, 0\right): C \in \mathbb{R}, \quad s \in \mathbb{R}^{3}\right\} .
$$

Hence, (4.9) implies that

$$
K(S):=\mathscr{K}(S) \cap N_{S} \mathscr{S}=\left\{\left(C \psi_{\alpha}, 0,0\right): C \in \mathbb{R}\right\}
$$


by (2.7) and (2.8). Therefore,

$$
\mathscr{K}(S) \cap N_{S} \mathscr{S} \cap T_{S} \mathscr{M}=K(S) \cap T_{S} \mathscr{M}=(0,0,0),
$$

since the vector $\left(\psi_{\alpha}, 0,0\right)$ is orthogonal to $T_{S} \mathscr{M}$ by (4.19). Now (4.20) follows since $E^{\prime \prime}(S) \geq 0$ by (4.6).

ii) Necessity. If the Wiener condition (1.13) fails, the null space $\mathscr{K}(S)$ is given by (4.16). Hence, (4.21) implies that now

$$
K(S)=\left\{\left(C \psi_{\alpha}, v, 0\right): C \in \mathbb{R}, v \in V\right\} .
$$

However, $\left(\psi_{\alpha}, \psi_{\alpha}\right)>0$. Hence, (4.19) implies that $\left(\psi_{\alpha}, v, 0\right) \notin T_{S} \mathscr{M}$ and the intersection

$$
\left.K(S) \cap T_{S} \mathscr{M}=\{0, v, 0): \quad v \in V\right\}
$$

is the nontrivial subspace of the dimension $d>0$. Thus, the Hessian $E^{\prime \prime}(S)$ vanishes on this nontrivial subspace of $N_{S} \mathscr{S} \cap T_{S} \mathscr{M}$.

Remark 4.6. The positivity of type (4.20) breaks down for the submanifold $\mathscr{S}(r):=\left\{S_{\alpha, r}: \alpha \in\right.$ $[0,2 \pi]\}$ with a fixed $r \in T_{N}$ instead of the solitary manifold $\mathscr{S}$. Indeed, in this case the corresponding tangent space is smaller,

$$
T_{S} \mathscr{S}(r)=\left\{\left(i C \psi_{\alpha}, 0,0\right): C \in \mathbb{R}\right\},
$$

and hence, the normal subspace $N_{S} \mathscr{S}(r)$ is larger, containing all vectors $(0, \bar{s}, 0)$ generating the shifts of the torus. However, all these vectors also belong to the null space (4.9) and to $T_{S} \mathscr{M}$. Respectively, the null space of the Hessian $E^{\prime \prime}(S)$ in $T_{S} \mathscr{M} \cap N_{S} \mathscr{S}(r)$ is 3-dimensional.

\subsection{The orbital stability}

Here we prove our main result.

Theorem 4.7. Let the conditions (1.11), (1.13) and (1.1) hold, and $\mathscr{S}$ is the solitary manifold (1.19). Then for any $\varepsilon>0$ there exists $\delta=\delta(\varepsilon)>0$ such that for $X(0) \in \mathscr{M}$ with $d_{\mathscr{V}}(X(0), \mathscr{S})<\delta$ we have

$$
d_{\mathscr{V}}(X(t), \mathscr{S})<\varepsilon, \quad t \in \mathbb{R}
$$

for the corresponding solution $X(t) \in C(\mathbb{R}, \mathscr{V})$ to (1.2)-(1.4).

For the proof is suffices to check the lower energy estimate (1.21):

$$
E(X) \geq v d_{\mathscr{V}}^{2}(X, \mathscr{S}) \quad \text { if } \quad d_{\mathscr{V}}(X, \mathscr{S}) \leq \delta, \quad X \in \mathscr{M}
$$

with some $v, \delta>0$. This estimate implies Theorem 4.7, since the energy is conserved along all trajectories. First, we prove similar lower bound for the energy Hessian.

Lemma 4.8. Let all conditions of Theorem 4.7 hold. Then for each $S \in \mathscr{S}$

$$
\left\langle Y, E^{\prime \prime}(S) Y\right\rangle>v\|Y\|_{\mathscr{W}}^{2}, \quad Y \in N_{S} \mathscr{S} \cap T_{S} \mathscr{M}
$$

where $v>0$. 
Proof. It suffices to prove (4.29) for $S=\left(\psi_{0}, 0,0\right)$. Note that $E^{\prime \prime}(S)$ is not complex linear due to the integral in (1.9). Hence, we express the action of $E^{\prime \prime}(S)$ in $\psi_{1}(x):=\operatorname{Re} \psi(x)$ and $\psi_{1}(x):=\operatorname{Im} \psi(x)$ : by the formula (1.15) of [21],

$$
E^{\prime \prime}(S) Y=\left(\begin{array}{cccc}
2 H_{0}+4 e^{2} \psi_{0} G \psi_{0} & 0 & 2 L & 0 \\
0 & 2 H_{0} & 0 & 0 \\
2 L^{*} & 0 & T & 0 \\
0 & 0 & 0 & M^{-1}
\end{array}\right) Y \quad \text { for } \quad Y=\left(\begin{array}{c}
\psi_{1} \\
\psi_{2} \\
q \\
p
\end{array}\right)
$$

where $H_{0}:=-\frac{1}{2} \Delta$ as in (2.13), and $\psi_{0}$ denotes the operator of multiplication by the real function $\psi_{0}(x) \equiv \sqrt{Z}$. The operator $L$ corresponds to the matrix

$$
L(x, n):=e \psi_{0}(x) G \nabla \sigma(x-n): \quad x \in \mathbb{R}^{3}, n \in \Gamma_{N}
$$

by formula (3.3) of [21] and $T$ corresponds to the real matrix with the entries

$$
T\left(n-n^{\prime}\right):=-\left\langle G \nabla \otimes \nabla \sigma\left(x-n^{\prime}\right), \sigma(x-n)\right\rangle, \quad n, n^{\prime} \in \Gamma_{N}
$$

by formula (3.4) of [21] since the corresponding potential $\Phi_{0}=0$. Thus, $E^{\prime \prime}(S)$ is a finite-rank perturbation of the operator with the discrete spectrum on the torus $T_{N}$. Moreover, (4.20) implies that the minimal eigenvalue of $E^{\prime \prime}(S)$ is positive. Therefore, (4.29) follows.

The positivity (4.29) implies the lower energy estimate (4.28), since the higher-order terms in (4.1) are negligible by the following lemma.

Lemma 4.9. Let $\sigma(x)$ satisfy (1.1). Then the remainder (4.7) admits the estimate

$$
|R(S, Y)| \leq C\|Y\|_{\mathscr{W}}^{3} \quad \text { for } \quad\|Y\|_{\mathscr{W}} \leq 1 .
$$

Proof. It suffices to prove the estimates

$$
\left\|\sqrt{G} \rho^{(1)}\right\|_{L^{2}\left(T_{N}\right)} \leq C_{1}\|Y\|_{\mathscr{W}}, \quad\left\|\sqrt{G} \rho^{(2)}\right\|_{L^{2}\left(T_{N}\right)} \leq C_{2}\|Y\|_{\mathscr{W}}^{2} \quad \text { for } \quad\|Y\|_{\mathscr{W}} \leq 1 .
$$

Then (4.33) will follow from (4.7).

i) By (4.4) we have for $Y=(\varphi, \varkappa, p)$

$$
\sqrt{G} \rho^{(1)}=\sqrt{G} \sigma^{(1)}-2 e \sqrt{G} \psi_{\alpha}(x) \cdot \varphi(x) .
$$

The operator $\sqrt{G}$ is bounded in $L^{2}\left(\mathbb{R}^{3}\right)$ by the definition (2.1). Hence,

$$
\left\|\sqrt{G} \sigma^{(1)}\right\|_{L^{2}\left(T_{N}\right)} \leq C|\varkappa|
$$

by (4.4). Applying to the second term the Cauchy-Schwarz and Hausdorff-Young inequalities, we obtain

$$
\left\|\sqrt{G} \psi_{\alpha}(x) \cdot \varphi\right\|_{L^{2}\left(T_{N}\right)} \leq C\left[\sum_{\xi \in \Gamma_{N}^{*}} \frac{|\hat{\varphi}(\xi)|^{2}}{|\xi|^{2}}\right]^{1 / 2} \leq C\|\hat{\varphi}\|_{L^{4}\left(\Gamma_{N}^{*}\right)}\left[\sum_{\xi \in \Gamma_{N}^{*}}|\xi|^{-4}\right]^{1 / 2} \leq C\|\varphi\|_{L^{4 / 3}\left(T_{N}\right)}
$$


Hence, the first inequality (4.34) is proved.

ii) Now we prove the second inequality (4.34). By (4.5) we have for $Y=(\varphi, \varkappa, p)$

$$
\sqrt{G} \rho^{(2)}(x)=\sqrt{G} \sigma^{(2)}(x)-e \sqrt{G}|\varphi(x)|^{2} .
$$

Similarly to (4.36)

$$
\left\|\sqrt{G} \sigma^{(2)}\right\|_{L^{2}\left(T_{N}\right)} \leq C|\varkappa|^{2}
$$

Finally, denoting $\beta(x):=|\varphi(x)|^{2}$, we obtain similarly to (4.37)

$$
\begin{aligned}
\left\|\sqrt{G}|\varphi(x)|^{2}\right\|_{L^{2}\left(T_{N}\right)} & \leq C\left[\sum_{\xi \in \Gamma_{N}^{*}} \frac{|\hat{\beta}(\xi)|^{2}}{|\xi|^{2}}\right]^{1 / 2} \leq C\|\hat{\beta}\|_{L^{4}\left(\Gamma_{N}^{*}\right)}\left[\sum_{\xi \in \Gamma_{N}^{*}}|\xi|^{-4}\right]^{1 / 2} \\
& \leq C_{1}\|\beta\|_{L^{4 / 3}\left(T_{N}\right)}=C_{1}\|\varphi\|_{L^{8 / 3}\left(T_{N}\right)}^{2} \leq C_{2}\|\varphi\|_{H^{1}\left(T_{N}\right)}^{2}
\end{aligned}
$$

by the Sobolev embedding theorem. Now the lemma is proved.

\section{A Conservation laws}

We deduce the conservation laws (2.11) by the Galerkin approximations [28].

Definition A.1. i) $\mathscr{V}_{m}$ with $m \in \mathbb{N}$ denotes finite dimensional submanifold of $\mathscr{V}$ formed by

$$
\left(\sum_{k \in \Gamma_{N}^{*}(m)} C_{k} e^{i k x}, q, p\right), \quad q \in T_{N}^{\bar{N}}, \quad p \in \mathbb{R}^{3 \bar{N}}
$$

where $\Gamma_{N}^{*}(m):=\left\{k \in \Gamma_{N}^{*}: k^{2} \leq m\right\}$.

ii) $\mathscr{W}_{m}$ with $m \in \mathbb{N}$ denotes the finite dimensional linear subspace of $\mathscr{W}$ spanned by

$$
\left(\sum_{k \in \Gamma_{N}^{*}(m)} C_{k} e^{i k x}, \varkappa, v\right), \quad \varkappa \in \mathbb{R}^{3 \bar{N}}, \quad v \in \mathbb{R}^{3 \bar{N}} .
$$

Obviously, $\mathscr{V}_{1} \subset \mathscr{V}_{2} \subset \ldots$, the union $\cup_{m} \mathscr{V}_{m}$ is dense in $\mathscr{V}$, and $\mathscr{W}_{m}$ are invariant with respect to $H$ and $J$. Let us denote by $P_{m}$ the orthogonal projector $\mathscr{X} \rightarrow \mathscr{W}_{m}$. This projector is also orthogonal in $\mathscr{W}$. Let us approximate the system (2.2) by the finite dimensional Hamilton systems on the manifold $\mathscr{V}_{m}$,

$$
\dot{X}_{m}(t)=J E_{m}^{\prime}\left(X_{m}(t)\right), \quad t \in \mathbb{R},
$$

where $E_{m}:=\left.E\right|_{\mathscr{V}_{m}}$ and $X_{m}(t)=\left(\psi_{m}(t), q_{m}(t), p_{m}(t)\right) \in C\left(\mathbb{R}, \mathscr{V}_{m}\right)$. The equation (A.3) can be also written as

$$
\left\langle\dot{X}_{m}(t), Y\right\rangle=-\left\langle E^{\prime}\left(X_{m}(t)\right), J Y\right\rangle, \quad Y \in \mathscr{W}_{m} .
$$

This form of the equation (A.3) holds since $E_{m}:=\left.E\right|_{\mathscr{V}_{m}}$ and $\mathscr{W}_{m}$ is invariant with respect to $J$. Equivalently,

$$
\dot{X}_{m}(t)=H X_{m}(t)+P_{m} N\left(X_{m}(t)\right) .
$$

The Hamiltonian form guarantees the energy and charge conservation (2.11):

$$
E\left(X_{m}(t)\right)=E\left(X_{m}(0)\right), \quad Q\left(X_{m}(t)\right)=Q\left(X_{m}(0)\right), \quad t \in \mathbb{R} .
$$


Indeed, the energy conservation holds by the Hamiltonian form (A.3), while the charge conservation holds by the Noether theory $[2,15,18]$ due to the $U(1)$-invariance of $E_{m}$, see (2.12).

The equation (A.5) admits a unique local solution for every initial state $X_{m}(0) \in \mathscr{V}_{m}$ since the right hand side is locally bounded and Lipschitz continuous. The global solutions exist by (2.10) and the energy and charge conservation (A.6).

Finally, we take any $X(0) \in \mathscr{V}$ and choose a sequence

$$
X_{m}(0) \rightarrow X(0), \quad m \rightarrow \infty
$$

where the convergence holds in the metric of $\mathscr{V}$. Therefore,

$$
E\left(X_{m}(0)\right) \rightarrow E(X(0)), \quad Q\left(X_{m}(0)\right) \rightarrow Q(X(0)) .
$$

Hence, (A.6) and (2.10) imply the basic uniform bound

$$
R:=\sup _{m \in \mathbb{N}} \sup _{t \in \mathbb{R}}\left|X_{m}(t)\right|_{\mathscr{V}}<\infty .
$$

Therefore, (A.5) and Lemma 3.3 imply the second basic uniform bound

$$
\sup _{m \in \mathbb{N}} \sup _{t \in \mathbb{R}}\left\|\dot{X}_{m}(t)\right\|_{\mathscr{W}-1}<C(R),
$$

since the operator $H: \mathscr{W} \rightarrow \mathscr{W}^{-1}$ is bounded, and the projector $P_{m}$ is also a bounded operator in $\mathscr{W} \subset$ $\mathscr{W}^{-1}$. Hence, the Galerkin approximations $X_{m}(t)$ are uniformly Lipschitz-continuous with values in $\mathscr{V}^{-1}$ :

$$
\sup _{m \in \mathbb{N}} d_{\mathscr{V}-1}\left(X_{m}(t), X_{m}(s)\right) \leq C(R)|t-s|, \quad s, t \in \mathbb{R} .
$$

Let us show that the uniform estimates (A.9) and (A.11) provide a compactness of the Galerkin approximations and the conservation laws. Let us recall that $\mathscr{X}:=\mathscr{V}^{0}$ and $\mathscr{V}:=\mathscr{V}^{1}$.

Proposition A.2. Let (1.1) hold and $X(0) \in \mathscr{V}$. Then

i) There exists a subsequence $m^{\prime} \rightarrow \infty$ such that

$$
X_{m^{\prime}}(t) \stackrel{\mathscr{X}}{\longrightarrow} X(t), \quad m^{\prime} \rightarrow \infty, \quad t \in \mathbb{R},
$$

where $X(\cdot) \in C(\mathbb{R}, \mathscr{X})$.

ii) Every limit function $X(\cdot)$ is a solution to $(2.14)$, and $X(\cdot) \in C(\mathbb{R}, \mathscr{V})$.

iii) The conservation laws (2.11) hold.

Proof. i) The convergence (A.12) follows from (A.9) and (A.10) by the Dubinsky 'theorem on three spaces' [10] (Theorem 5.1 of [28]). Namely, the embedding $\mathscr{V} \subset \mathscr{X}$ is compact by the Sobolev theorem, and hence, (A.12) holds by (A.9) for $t \in D$, where $D$ is a countable dense set. Finally, let us use the interpolation inequality and (A.9), (A.11): for any $\varepsilon>0$

$$
d_{\mathscr{X}}\left(X_{m}(t), X_{m}(s)\right) \leq \varepsilon d_{\mathscr{V}}\left(X_{m}(t), X_{m}(s)\right)+C(\varepsilon) d_{\mathscr{V}-1}\left(X_{m}(t), X_{m}(s)\right) \leq 2 \varepsilon R+C(\varepsilon, R)|t-s|
$$

This inequality implies the equicontinuity of the Galerkin approximations with the values in $\mathscr{X}$. Hence, convergence (A.12) holds for all $t \in \mathbb{R}$ since it holds for the dense set of $t \in D$. The same 
equicontinuity also implies the continuity of the limit function $X \in C(\mathbb{R}, \mathscr{X})$.

ii) Integrating equation (A.5), we obtain

$$
\int_{0}^{t}\left\langle\dot{X}_{m}(t), Y\right\rangle d s=\int_{0}^{t}\left\langle X_{m}(s), H Y\right) d s+\int_{0}^{t}\left\langle N\left(X_{m}(s)\right), Y\right\rangle d s, \quad Y \in \mathscr{W}_{m}
$$

Below we will write $m$ instead of $m^{\prime}$. To prove (2.14) it suffices to check that in the limit $m \rightarrow \infty$, we get

$$
\int_{0}^{t}\langle\dot{X}(t), Y\rangle d s=\int_{0}^{t}\langle X(s), H Y\rangle d s+\int_{0}^{t}\langle N(X(s)), Y\rangle d s, \quad Y \in \mathscr{W}_{n}, \quad n \in \mathbb{N} .
$$

The convergence of the left hand side and of the first term on the right hand side of (A.14) follow from (A.12) and (A.7) since $H Y \in \mathscr{W}_{m}$.

It remains to consider the last integral of (A.14). The integrand is uniformly bounded by (A.9) and Lemma 3.3. Hence, it suffices to check the pointwise convergence

$$
\left\langleN ( X _ { m } ( t ) , Y \rangle \longrightarrow \left\langle N(X(t), Y\rangle, \quad m \rightarrow \infty, \quad Y \in \mathscr{W}_{n}\right.\right.
$$

for any $t \in \mathbb{R}$. Here $N\left(X_{m}(t)\right)=\left(i e \Phi_{m}(t) \psi_{m}(t), p_{m}(t), f_{m}(t)\right)$ according to the notations (2.15), and $Y=(\varphi, \varkappa, v) \in \mathscr{W}_{n}$. Hence, (A.16) reads

$$
i e\left(\Phi_{m}(t) \psi_{m}(t), \varphi\right)+p_{m}(t) \varkappa+f_{m}(t) v \rightarrow i e(\Phi(t) \psi(t), \varphi)+p(t) \varkappa+f(t) v, \quad m \rightarrow \infty .
$$

The convergence of $p_{m}(s) \varkappa$ follows from (A.12) (with $m^{\prime}=m$ ). To prove the convergence of two remaining terms, we first show that

$$
\Phi_{m}(t):=G \rho_{m} \stackrel{C\left(T_{N}\right)}{\longrightarrow} \Phi(t):=G \rho, \quad m \rightarrow \infty .
$$

Indeed, (A.12) implies that

$$
\psi_{m}(t) \stackrel{L^{2}\left(T_{N}\right)}{\longrightarrow} \psi(t), \quad q_{m}(t) \rightarrow q(t), \quad m \rightarrow \infty .
$$

The sequence $\psi_{m}(t)$ is bounded in $H^{1}\left(T_{N}\right)$ by (A.9). Hence, $\psi(t) \in H^{1}\left(T_{N}\right)$ and the sequence $\rho_{m}(t)$ is bounded in the Sobolev space $W^{1,3 / 2}\left(T_{N}\right)$ by (3.4). Therefore, the sequence $\rho_{m}(t)$ is precompact in $L^{2}\left(T_{N}\right)$ by the Sobolev compactness theorem. Hence,

$$
\rho_{m} \stackrel{L^{2}\left(T_{N}\right)}{\longrightarrow} \rho, \quad m \rightarrow \infty
$$

by (A.19). Therefore, (A.18) holds since the operator $G: L^{2}\left(T_{N}\right) \rightarrow C\left(T_{N}\right)$ is continuous. From (A.18) and (A.19) it follows that

$$
\Phi_{m}(t) \psi_{m}(t) \stackrel{L^{2}\left(T_{N}\right)}{\longrightarrow} \Phi(t) \psi(t), \quad f_{m}(t) \rightarrow f(t), \quad m \rightarrow \infty
$$

which proves (A.17). Now (A.15) is proved for $Y \in \mathscr{V}_{n}$ with any $n \in \mathbb{N}$. Hence, $X(t)$ is a solution to (2.2). Finally, $\|N(X(\cdot))\|_{\mathscr{W}}$ is a bounded function by (A.9) and Lemma 3.3. Hence, (2.14) implies that $X(\cdot) \in C(\mathbb{R}, \mathscr{V})$. 
iii) The conservation laws (A.6) and the convergences (A.7), (A.12) imply that

$$
E(X(t)) \leq E(X(0)), \quad Q(X(t)) \leq Q(X(0)), \quad t \in \mathbb{R} .
$$

The last inequality holds by the first convergence of (A.19). The first inequality follows from the representation

$$
E\left(X_{m}(t)\right)=\frac{1}{2}\left\|\nabla \psi_{m}(t)\right\|_{L^{2}\left(T_{N}\right)}^{2}+\frac{1}{2}\left\|\sqrt{G} \rho_{m}(t)\right\|_{L^{2}\left(T_{N}\right)}^{2}+\sum_{n \in \Gamma_{n}} \frac{p_{m}^{2}(n, t)}{2 M} .
$$

Namely, the last two terms on the right hand side converge by (A.20) and (A.12). Moreover, the first term is bounded by (A.9). Hence, the first convergence of (A.19) implies the weak convergence

$$
\nabla \psi_{m}(t) \stackrel{L_{w}^{2}\left(T_{N}\right)}{\longrightarrow} \nabla \psi(t)
$$

by the Banach theorem. Now the first inequality of (A.22) follows by the property of the weak convergence in the Hilbert space. Finally, the opposite inequalities to (A.22) are also true by the uniqueness of solutions $X(\cdot) \in C(\mathbb{R}, \mathscr{V})$, which is proved in Proposition 3.2.

\section{B Jellium ground states}

We describe all solutions to (1.2) - (1.4) with minimal energy (1.9), give some examples of ion densities illustrating the Jellium and the Wiener conditions, and show the existence of non-periodic ground states.

\section{B.1 Description of all ground states}

The following lemma gives the description of all ground states.

Lemma B.1. Let the Jellium condition (1.11) hold. Then all solutions to (1.2)-(1.4) of minimal (zero) energy are $\left(\psi_{\alpha}, q^{*}, 0\right)$ with $q^{*} \in T_{N}{ }^{N}$ satisfying the identity

$$
\sum_{n \in \Gamma_{N}} \sigma\left(x-q^{*}(n)\right) \equiv e Z, \quad x \in T_{N}
$$

Proof. First, let us note that the $\Gamma_{N}$-periodic solutions (1.16) have the zero energy, and the identity (B.1) holds for $q^{*}=\bar{r}$ by (1.12).

Further, for any solution with zero energy (1.9) all summands on the right hand side of (1.9) vanish. The first integral vanishes only for constant functions. Hence, the normalization condition (1.7) gives

$$
\psi(x, t) \equiv \psi_{\alpha(t)}(x) \equiv e^{i \alpha(t)} \sqrt{Z}, \quad \alpha(t) \in \mathbb{R} .
$$

Then

$$
\rho^{e}(x, t):=-e\left|\psi_{\alpha(t)}(x)\right|^{2} \equiv-e Z, \quad x \in T_{N}, t \in \mathbb{R},
$$

similarly to (1.18). Further, the second summand of (1.9) vanishes only for $\rho(x, t) \equiv 0$ that is equivalent to (B.1) with $q(n, t)$ instead of $q^{*}(n)$ by (B.3). However, $\partial_{t} q(n, t)=p(n, t) \equiv 0$ for the zero energy (1.9). Hence,

$$
q(n, t) \equiv q^{*}(n), \quad t \in \mathbb{R},
$$

where $q^{*}$ satisfies (B.1). Moreover, $\Phi(x, t) \equiv 0$ by the Poisson equation (1.3) with $\rho(x, t) \equiv 0$. Hence, finally, substituting (B.2) into (1.2) with $\Phi(x, t) \equiv 0$, we obtain that $\alpha(t) \equiv$ const.

This lemma implies that all $\Gamma_{N}$-periodic ground states are given by (1.16). 


\section{B.2 Jellium and Wiener conditions. Examples}

The Wiener condition (1.13) for the ground states (1.16) holds under the generic assumption (1.15). On the other hand, (1.13) does not hold for the simplest Jellium model, when $\sigma(x)$ is the characteristic function

$$
\sigma(x)=\sigma_{1}(x):=\left\{\begin{array}{ll}
e Z, & x \in \Pi \\
0, & x \in T_{N} \backslash \Pi
\end{array} \mid\right.
$$

Indeed, in this case the Fourier transform

$$
\hat{\sigma}_{1}(\xi)=e Z \hat{\chi}_{1}\left(\xi_{1}\right) \hat{\chi}_{1}\left(\xi_{2}\right) \hat{\chi}_{1}\left(\xi_{3}\right) ; \quad \hat{\chi}_{1}(s)=\frac{2 \sin s / 2}{s}, \quad s \in \mathbb{R} \backslash 0,
$$

where $\chi_{1}(s)$ is the characteristic function of the interval $[0,1]$. In this case we have for $\theta=\left(0, \theta_{2}, \theta_{3}\right)$,

$$
\Sigma(\theta)=\sum_{m \in \mathbb{Z}^{3}: m_{1}=0}\left[\frac{\xi \otimes \xi}{|\xi|^{2}}|\hat{\sigma}(\xi)|^{2}\right]_{\xi=\theta+2 \pi m}
$$

which is a degenerate matrix since $\xi_{1}=0$ in each summand. Hence, (1.13) fails. Similarly, the Wiener condition fails for $\sigma_{k}(x)=e Z \chi_{k}\left(x_{1}\right) \chi_{k}\left(x_{2}\right) \chi_{k}\left(x_{3}\right)$ where $\chi_{k}=\xi * \ldots * \xi$ ( $k$ times) with $k=2,3, \ldots$, since in this case

$$
\hat{\sigma}_{k}(\xi)=e Z \hat{\chi}_{k}\left(\xi_{1}\right) \hat{\chi}_{k}\left(\xi_{2}\right) \hat{\chi}_{k}\left(\xi_{3}\right) ; \quad \hat{\chi}_{k}(s)=\left[\frac{2 \sin s / 2}{s}\right]^{k}, \quad s \in \mathbb{R} \backslash 0 .
$$

\section{B.3 Non-periodic ground states}

It is easy to construct ground states which are not $\Gamma_{1}$-periodic in the case of characteristic function (B.5). Namely, the identity (B.1) obviously holds for periodic arrangement of ions (1.17). Now let us modify this periodic arrangement as follows:

$$
q^{*}(n)=\left(r_{1}, r_{2}, r_{3}+\tau\left(n_{1}, n_{2}\right)\right), \quad n \in \Gamma_{N},
$$

where $\tau\left(n_{1}, n_{2}\right)$ is an arbitrary point of the circle $\mathbb{R} / N \mathbb{R}$. Now (B.1) obviously holds for any arrangement of ions (B.9).

Next lemma gives a more general spectral assumptions on $\sigma$ which provide ground states with non-periodic ion arrangements. For example, let us assume that

$$
\sigma(\xi)=0, \quad \xi_{3} \in 2 \pi \mathbb{Z} \backslash 0, \quad \text { and } \quad \sigma\left(\xi_{1}, \xi_{2}, 0\right)=0, \quad\left(\xi_{1}, \xi_{2}\right) \in 2 \pi \mathbb{Z}^{2} \backslash 0 .
$$

In particular, this holds for the densities (B.8) with all $k=1, \ldots$

Lemma B.2. Let $\sigma$ satisfy the spectral condition (B.10). Then there exist ground states which are not $\Gamma_{1}$-periodic.

Proof. In the Fourier transform (B.1) reads

$$
\int_{T_{N}} e^{i \xi x} \sum_{n \in \Gamma_{N}} \sigma\left(x-n-q^{*}(n)\right) d x=\hat{\sigma}(\xi) \sum_{n \in \Gamma_{N}} e^{i \xi(n+q(n))}=\left\{\begin{aligned}
e Z \bar{N}, & \xi=0 \\
0, & \xi \in \Gamma_{N}^{*} \backslash 0 .
\end{aligned}\right.
$$


These identities hold for any density $\sigma$ satisfying (1.11) if

$$
\sum_{n \in \Gamma_{N}} e^{i \xi\left(n+q^{*}(n)\right)}=0, \quad \xi \in \Gamma_{N}^{*} \backslash \Gamma_{1}^{*} .
$$

In particular, $q^{*}=\bar{r}$ satisfies the system (B.12) since then

$$
\sum_{n \in \Gamma_{N}} e^{i \xi\left(n+q^{*}(n)\right)}=\sum_{n \in \Gamma_{N}} e^{i \xi(n+r)}=e^{i \xi r} \sum_{n \in \Gamma_{N}} e^{i \xi n}=0, \quad \xi \in \Gamma_{N}^{*} \backslash \Gamma_{1}^{*} .
$$

Indeed,

$$
\sum_{n \in \Gamma_{N}} e^{i \xi n}=\sum_{n \in \Gamma_{N}} e^{i\left(\xi_{1} n_{1}+\xi_{2} n_{2}+\xi_{3} n_{3}\right)}=\sum_{n_{1}=0}^{N-1} e^{i \xi_{1} n_{1}} \sum_{n_{2}=0}^{N-1} e^{i \xi_{2} n_{2}} \sum_{n_{3}=0}^{N-1} e^{i \xi_{3} n_{3}}=0
$$

since at least one $\xi_{k} \notin 2 \pi \mathbb{Z}$ for $\xi \in \Gamma_{N}^{*} \backslash \Gamma_{1}^{*}$. Now we modify $\bar{r}$ as follows:

$$
\left.q^{*}(n):=\left(a_{1}\left(n_{1}, n_{2}\right)\right), a_{2}\left(n_{1}, n_{2}\right), r_{3}\right), \quad n \in \Gamma_{N},
$$

where $\left.a_{1}\left(n_{1}, n_{2}\right)\right)$ and $a_{2}\left(n_{1}, n_{2}\right)$ are arbitrary points of the circle $R / 2 \pi \mathbb{Z}$. Then for $\xi \in \Gamma_{N}^{*} \backslash \Gamma_{1}^{*}$ we have

$$
\sum_{n \in \Gamma_{N}} e^{i \xi\left(n+q^{*}(n)\right)}=\sum_{n_{1}, n_{2}=0}^{N-1} e^{i\left(\xi _ { 1 } \left(n_{1}+a_{1}\left(n_{1}, n_{2}\right)+\xi_{2}\left(n_{2}+a_{2}\left(n_{1}, n_{2}\right)\right.\right.\right.} \sum_{n_{3}=0}^{N-1} e^{i \xi_{3} n_{3}}=0 \quad \text { if } \quad \xi_{3} \notin 2 \pi \mathbb{Z} .
$$

Hence, all identities (B.11) hold by (B.10).

\section{B.4 On the problem of periodicity}

In the non-periodic examples above the Wiener condition fails. We suppose that the Wiener condition provides only periodic ground states, however this is an open problem. For densities $\sigma$ satisfying a more strong condition (1.13), the identity (B.1) is equivalent to the system (B.12) by (B.11). The system (B.12) can be written as an 'algebraic system'

$$
\sum_{n \in \Gamma_{N}} w_{1}^{m_{1}}(n) w_{2}^{m_{2}}(n) w_{3}^{m_{3}}(n)=0, \quad m \in \mathbb{Z}^{3} \backslash N Z^{3} .
$$

for

$$
w_{j}\left(n_{1}, n_{2}, n_{3}\right):=e^{i \frac{2 \pi}{N}\left[n_{j}+q_{j}^{*}(n)\right]}, \quad n \in \Gamma_{N}, \quad j=1,2,3 .
$$

The $\Gamma_{N}$-periodicity of $q^{*}$ is equivalent to the fact that only solutions are

$$
w_{j}\left(n_{1}, n_{2}, n_{3}\right)=C_{j} \lambda^{n_{j}}, \quad j=1,2,3,
$$

where $\lambda:=e^{i \frac{2 \pi}{N}}$. For the corresponding 1D analog

$$
\sum_{n_{1}=0}^{N-1} w_{1}^{m_{1}}\left(n_{1}\right)=0, \quad m=1, \ldots, N-1,
$$

the only solutions are $w_{1}\left(n_{1}\right):=C_{1} \lambda^{n_{j}}$ that follows easily from the Newton-Girard formulas guessed by Girard in 1629 and rediscovered (without a proof) by Newton in 1666. The formulas were proved by Euler in 1747 , see [13]. 


\section{References}

[1] R.A. Adams, Sobolev Spaces, Academic Press, NY, 1975.

[2] V. Arnold, Mathematical Methods of Classical Mechanics, Springer, New York, 1978.

[3] F. Bonetto, J. L. Lebowitz, L. Rey-Bellet, Fourier's law: a challenge to theorists, p. 128-150 in: Fokas, A. (ed.) et al., Mathematical physics 2000. International congress, London, GB, 2000, Imperial College Press, London, 2000.

[4] M. Born, K. Huang, Dynamical Theory of Crystal Lattices, The Clarendon Press, Oxford University Press, New York, 1998.

[5] E. Cancès, S. Lahbabi, M. Lewin, Mean-field models for disordered crystals, J. Math. Pures Appl. (9) 100 (2013), no. 2, 241-274.

[6] E. Cancès, G. Stoltz, A mathematical formulation of the random phase approximation for crystals, Ann. I. H. Poincaré - AN 29 (2012), 887-925.

[7] L. Catto, C. Le Bris, P.-L. Lions, The Mathematical Theory of Thermodynamic Limits: ThomasFermi Type Models, Clarendon Press, Oxford, 1998.

[8] L. Catto, C. Le Bris, P.-L. Lions, On the thermodynamic limit for Hartree-Fock type models, Ann. Inst. Henri Poincaré, Anal. Non Linéaire 18 (2001), no. 6, 687-760.

[9] L. Catto, C. Le Bris, P.-L. Lions, On some periodic Hartree-type models for crystals, Ann. Inst. Henri Poincaré, Anal. Non Linéaire 19 (2002), no. 2, 143-190.

[10] Yu. A. Dubinsky, Weak convergence in non-linear elliptic and parabolic equations, Mat. USSR Sb. 67 (4) (1965), 609-642 (in Russian).

[11] F.J. Dyson, Ground-state energy of a finite system of charged particles, J. Math. Phys. 8, 15381545 (1967).

[12] F.J. Dyson, A. Lenard, Stability of matter I, J. Math. Phys. 8 (1967), 423-434; II, ibid. 9 (1968), 698-711.

[13] L. Euler, Demonstratio gemina theorematis Newtoniani, quo traditur relatio inter coefficientes cuiusvis aequiationis algebraicae et summas potestatum radicum eiusdem, Opuscula varii argumenti 2 (1750), 108-120. (English translation by J. Bell: A double demonstration of a theorem of Newton, which gives a relation between the coefficients of an algebnraic equation and the sums of the powers of its roots, arXiv:0707.0699 [math.HO])

[14] G. Giuliani, G. Vignale, Quantum Theory of the Electron Liquid, Cambridge University Press, Cambridge, 2005.

[15] M. Grillakis, J. Shatah, W. Strauss, Stability theory of solitary waves in the presence of symmetry. I, J. Funct. Anal. 74 (1987), 160-197.

[16] L. Hörmander, The Analysis of Linear Partial Differential Operators. I. Distribution Theory and Fourier Analysis, Springer, Berlin, 2003. 
[17] C. Kittel, Introduction to Solid State Physics, Wiley \& Sons, Hoboken, NJ, 2005.

[18] A. I. Komech, Quantum Mechanics: Genesis and Achievements, Springer, Dordrecht, 2013.

[19] A. I. Komech, On crystal ground state in the Schrödinger-Poisson model, SIAM J. Math. Anal. 47 (2015), no. 2, 1001-1021. arXiv:1310.3084

[20] A. I. Komech, On crystal ground state in the Schrödinger-Poisson model with point ions, Math. Notes 99 (2016), no. 6, 886-894. arXiv:1409.1847

[21] A. Komech, E. Kopylova, On the linear stability of crystals in the Schrödinger-Poisson model, accepted in J. Stat. Phys., 2016. arXiv:1505.07074

[22] C. Le Bris, P.-L. Lions, From atoms to crystals: a mathematical journey, Bull. Am. Math. Soc., New Ser. 42 (2005), no. 3, 291-363.

[23] J.L. Lebowitz, E.H. Lieb, Existence of thermodynamics for real matter with Coulomb forces, Phys. Rev. Lett. 22 (13) (1969), 631-634.

[24] J.L. Lebowitz, E.H. Lieb, Lectures on the thermodynamic limit for Coulomb systems, in: Springer Lecture Notes in Physics, Vol. 20, Springer, 1973, pp. 136-161.

[25] M. Lewin, J. Sabin, The Hartree equation for infinitely many particles. I. Well-posedness theory, arXiv:1310.0603.

[26] M. Lewin, J. Sabin, The Hartree equation for infinitely many particles. II. Dispersion and scattering in 2D, arXiv:1310.0604.

[27] E.H. Lieb, R. Seiringer, The Stability of Matter in Quantum Mechanics, Cambridge University Press, Cambridge, 2009.

[28] J.-L. Lions, Quelques méthodes de résolution des problèmes aux limites non linéaires, Dunod; Gauthier-Villars, Paris, 1969.

[29] J.A. Stratton, Electromagnetic Theory, John Wiley \& Sons, Inc., Hoboken, New Jersey, 2007.

[30] M. Ziman, The Calculation of Bloch Functions, Academic Press, NY, 1971. 